\title{
Driver's warning notifications by using FM RDS technology
}

\author{
Florin Doru HUŢU* and Radu Gabriel BOZOMITU ${ }^{\dagger}$ \\ *Univ Lyon, INSA Lyon, Inria, CITI, EA3720, 69621 Villeurbanne, France \\ ${ }^{\dagger}$ Faculty of Electronics, Telecommunications and Information Technology, \\ "Gheorghe Asachi" Technical University, Iaşi 700050, Romania \\ Email: florin-doru.hutu@insa-lyon.fr
}

\begin{abstract}
In this paper, the use of Software Defined Radio (SDR) transceivers for broadcasting drivers' warning notifications is tackled. More precisely, by employing the Radio Data System (RDS) protocol of a local Frequency Modulation (FM) transmission, road traffic's dangerous areas (rail crossing, unmarked crossroads, etc.) are announced. The code of a warning message is broadcasted in the close vicinity of the dangerous area and, at the receiver side, a corresponding image is displayed on the car's tablet PC. In order to study the coexistence of such kind of transmissions with the regional or national ones, the performance in terms of bit error rate (BER) with respect to the difference between the two received power levels is studied. Then, based on a simplified channel model, this paper gives a method to estimate the local FM transmission range, able to cover the dangerous area without transmission errors on the RDS part.
\end{abstract}

\section{INTRODUCTION}

More than ten years ago, the European Union had the ambition to halve the number of road fatalities by 2020 with respect to the values of 2010. Unfortunately, this target was not reached, and in 2019 the EU average was 51 road fatalities per million inhabitants. In the same time, Romania, with 96 fatalities per million inhabitants, had the most dangerous roads in the European Union. France, with the 50 fatalities per million inhabitants was representative for the EU average and Iceland had the safest roads in 2019 (17 fatalities per million inhabitants[1].

For Romania, this situation can be explained by the tremendous increase of the car fleet in the last two decades (from 3 million to 8 million vehicles) while the road infrastructure did not keep pace with this evolution. In this context, the implementation of solutions able to increase traffic safety remains a stringent problem.

Many car accidents are due to the occurrence of sudden dangerous situations in traffic for which the drivers are not prepared to react or they are reacting too slowly. In the literature, several solutions, based on the wireless transmission of drivers' notifications, are proposed. Among them, the authors of [2] are proposing to broadcast driver's warning notifications through the FM radio infrastructure by the means of pictograms sent through Quadrature Phase Shift Keying (QPSK) modulation on the monophonic component of the multiplex (MPX) signal.

Different other types of radio access technologies, such as cellular vehicle-to-everything $(\mathrm{C}-\mathrm{V} 2 \mathrm{X})$ and dedicated short- range communication (DSRC) are used in order to implement vehicular networks for robust communications among vehicles. By using these communication technologies, many applications like road traffic safety together with infotainment related services developed within new intelligent transportation systems (ITS) are envisaged in the near future. DSRC and CV2X are technologies employed for very high-speed (Mbps) and at high-frequency $(5.9 \mathrm{GHz})$ data exchange and require extensive and very expensive infrastructure deployment. The range of DSRC is typically 300 meters, while $\mathrm{C}-\mathrm{V} 2 \mathrm{X}$ may have $20-30 \%$ more range than DSRC as well as significant improvement in terms of performance when obstructions between emitters and receivers occurs [3].

The classical RDS system provides the TA (traffic announcements) flag which may be set up in order to receive national or regional announcements about traffic events. When TA is set, the radio receiver is tuned to a special FM channel where flash information is broadcasted. Another example of classic traffic broadcasting service is the TMC (Traffic Message Channel). Once again, regional or national traffic information is broadcasted and generally integrated directly into the car's navigation system.

In this context, our paper proposes a local warning system through image notifications based on FM radio transmissions. More precisely, the drivers are receiving notifications from the local FM emitters which are deployed in the close vicinity of an imminent danger (rail crossing, unmarked crossroads, etc.). These notifications are transmitted as codes sent on the RDS (Radio Data System) component and these codes are associated to pictograms which are selected from a database stored on the car's tablet PC. As soon as the code is received, the pictogram is displayed on the tablet PC's screen and an audio notification may be also be played back.

The rest of the paper is organized as follows: in section 2, the scenario of transmitting warning notifications by the means of local FM emitters is detailed. Through simulation results, section 3 presents the impact of the classical FM broadcasting on the local FM transmission. More precisely, the coexistence of the two kind of FM emissions (classical and local ones) is studied and the local RDS signal's transmission quality is tackled. The bit error rate was chosen as indicator of the local FM transmission's communication quality. 


\section{Problem Statement}

In this paper, the scenario of using local FM emitters in order to transmit drivers' warning notifications is studied. These FM transmissions are performed with a relatively high power level in order to overcome the existing broacasting of a classical FM station emitting on the same channel. The range of this FM transmission need to be long enough such that the driver adapts its driving with respect of the imminent danger. More precisely, the code transmitted on the RDS part need be decoded properly and the corresponding image displayed in a short time.

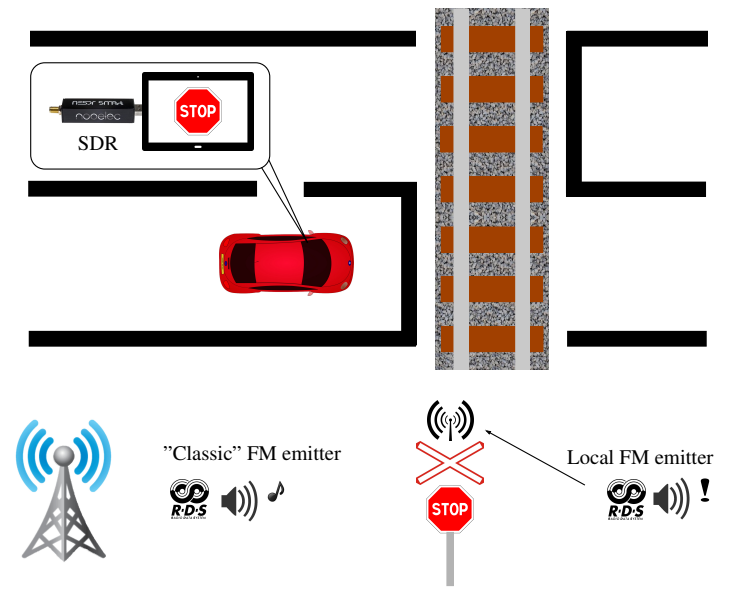

Fig. 1. Scenario of a driver's warning notification strategy by using local FM emitters. The local FM emitter is installed at the road sign level and the emission power is high enough to assure a zero BER for the RDS signal in the close vicinity.

For the proposed solution, several advantages can be listed. The main one is that the solution is entirely compatible with the existing car lot. In the absence of a tablet PC, the driver is notified either by the audio signal, either by a text notification, corresponding to the RDS transmission. Another advantage is that the FM radio broadcasting infrastructure is a mature technology, widespread in all European countries and especially in Romania. Consequently, the deployment of the proposed solution is relatively inexpensive.

As can be seen from Figure 2, the classical FM composite signals have two distinct parts. First, the MPX (multiplex component) part is the one generated by a stereo encoder based on the two audio channels. Second, the RDS part is dedicated to broadcast, in a digital form, a certain amount of information. A classical commercial radio receiver, compliant to the RDS standard [4], can receive static or dynamic information like the name of the radio station, the name or the type the broadcasted program, etc. On the physical layer, after passing through a differential encoder, the RDS data is transposed around a $57 \mathrm{kHz}$ subcarrier by using the PSK (Phase Shift Keying) modulation. The RDS data is sent with a symbol rate of 1187.5 syms/sec.

According to the recommendations of the ITU (International Telecommunications Union) [5], the composite signal

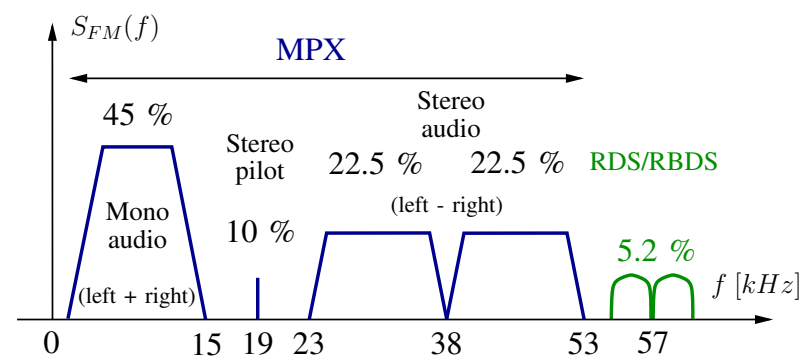

Fig. 2. Spectrum of a classical FM composite signal. The percentages are corresponding to the contribution of each component to the composite signal's power level.

presented on Figure 2 modulates an RF carrier frequency which may vary from $87.5 \mathrm{MHz}$ up to $108 \mathrm{MHz}$. In order to bring immunity with respect to the amplitude level fluctuation, the frequency modulation with a maximal deviation of \pm 75 $\mathrm{kHz}$ is employed. According to FM standards, this frequency deviation corresponds to a channel bandwidth of $200 \mathrm{kHz}$.

The FM emissions are performed with a relatively high power level in order to cover large areas. According to [6], the average levels of the received electrical fields in order to obtain a satisfactory FM reception are function of the scenario. Table I is gathering these values together with the corresponding power levels, when the far field condition is satisfied and for a receiving antenna having $5 \mathrm{dBi}$ gain. The considered RF frequency is $100 \mathrm{MHz}$.

\begin{tabular}{|c||c|c|c|}
\hline Area & Rural & Urban & Large cities \\
\hline $\mathbf{E}[\mathbf{d B} \mu \mathbf{V} / \mathbf{m}]$ & 54 & 66 & 74 \\
\hline $\mathrm{P}[\mathrm{dBm}]$ & -58.21 & -46.21 & -38.21 \\
\hline
\end{tabular}

TABLE I

MEDIAN FIELD STRENGTH AND THE CORRESPONDING RECEIVED POWER LEVELS FOR DIFFERENT FM RECEPTION SCENARIOS

As stated, the emitters installed in the close vicinity of the imminent danger are broadcasting warning signals on a FM channel through the RDS component. The power level of the local notification signal can be approximated if the following scenario is considered. The antenna of the local FM emitter is considered placed at a height $H=3.5$ meters, an average value of the road sign's height. The antennas installed on the car's rooftop and at the local emitter level are omnidirectional ones. Moreover, a ground plane is considered at the car's aerial level and its height is $h=1.5$ meters, an average value for a car's height.

Under these conditions, by employing the Friis formula in free space [7], the received power level $\left(P_{R}\right)_{d B m}$ can be written as in equation (1):

$$
\begin{aligned}
& P_{r}=E I R P+G_{R}-22-20 \cdot \log _{10}\left(\frac{d}{\lambda_{0}}\right)+ \\
& +20 \cdot \log _{10}\left[\left(\frac{\Delta h^{2}+d^{2}}{d^{2}}\right) \cos ^{2}\left(\frac{\pi}{2} \cdot \frac{\Delta h}{\sqrt{\Delta h^{2}+d^{2}}}\right)\right]
\end{aligned}
$$


where $d$ represents the distance between the two antennas, $\left(G_{R}\right)_{d B i}$ is the gain of the receiving antenna, $\Delta h=H-h$ represents the height difference between the two antennas and $\lambda_{0}$ is the free space wavelength.

Figure 3 presents the received power level variation with respect to the distance $d$ between the two antennas when the receiving antenna's maximum gain is $5 \mathrm{dBi}$. The local emitter's EIRP (equivalent isotropic radiated power) is arbitrarily chosen at $10 \mathrm{dBm}$ and the RF frequency $f=100 \mathrm{MHz}$.

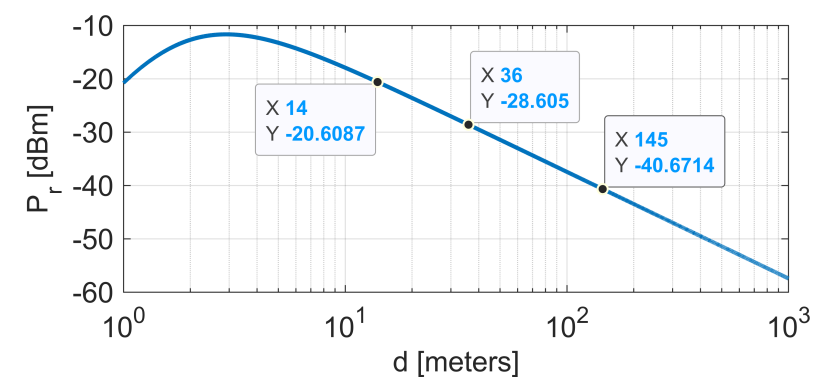

Fig. 3. Local FM signal's power variation with respect to the distance $d$ between the car's aerial and the local FM emitter's antenna.

By taking into account this variation of the local's FM signal strength and the average received signal strength for the national/regional FM transmissions (Table I), the range of the local FM transmission such that the RDS transmission's BER remains insignificant can be estimated.

\section{SimULATION RESULTS}

In order to estimate the BER variation, a simulation model based on the software-defined radio concept was implemented in Matlab - Simulink, including a stereo FM transmitter and receiver using the RDS standard. An additive white Gaussian noise (AWGN) is also considered in order to bring more realism to the communication channel.

In practice, the local FM signal may be obtained by translating the baseband composite part on a carrier frequency $(87.5-108 \mathrm{MHz})$ that has very good propagation properties by using a professional transmitter as for example an USRP (Universal Software Radio Peripheral) device. In our model, in order to be able to perform the simulations without exceeding the processing capabilities of the computing unit, the carrier frequency was set to $1 \mathrm{MHz}$.

The simulation setup includes two FM transmitters emitting on the same RF frequency, one for broadcasting the FM high priority local warning signal $\left(s_{R F L}(t)\right)$ and having a power level $P_{R F L}$, and the other one for transmitting the FM signal generated by the national/regional station $\left(s_{R F N}(t)\right.$ having the power level $P_{R F N}$ ). The sampling frequency used in the proposed system was set to $228 \mathrm{kHz}$, which is a multiple of the $1187.5 \mathrm{~Hz}$, the data rate of the RDS signal.

The software defined FM receiver uses a non-coherent FM demodulator based on the complex delay line frequency discriminator employed in order to extract the MPX signal. It uses also a stereo FM demultiplexer, a stereo FM decoder to extract the audio stereo part by using blocks from the "RTLSDR book library" [8] and a physical layer RDS decoder [9].

As stated, in the vicinity of a traffic dangerous situation, the signal generated by the local FM transmitter need to have a higher power level compared to the signal generated by the national station, so that it can be received by traffic participants without errors. The model proposed in this paper takes into account the distortions introduced by the AWGN communications channel, as well as the distortions generated by the national/regional station, which acts as noise for the local FM transmitter.

The distortions introduced by the AWGN communication channel have been modeled by considering different values for the received RDS signal to noise ratio $\left(S_{R D S} / N\right)$. More precisely, three transmission scenarios, corresponding to different values of the $S_{R D S} / N$ ratio $(8 \mathrm{~dB}, 11 \mathrm{~dB}$ and $14 \mathrm{~dB})$ have been analyzed in the paper. In our setup, the transmission of the local FM station without the impact of the national FM one, corresponds to a transmission of the RDS signal without any errors. In this particular case, the signal to noise ration of the RDS component is higher than $S_{R D S} / N=11 \mathrm{~dB}$.

In Fig. 4, the spectrum of the received MPX high priority warning signal for an $S_{R D S} / N=11 \mathrm{~dB}$ and a power difference of $\Delta_{P}=P_{R F L}-P_{R F N}=18 \mathrm{~dB}$ is presented.

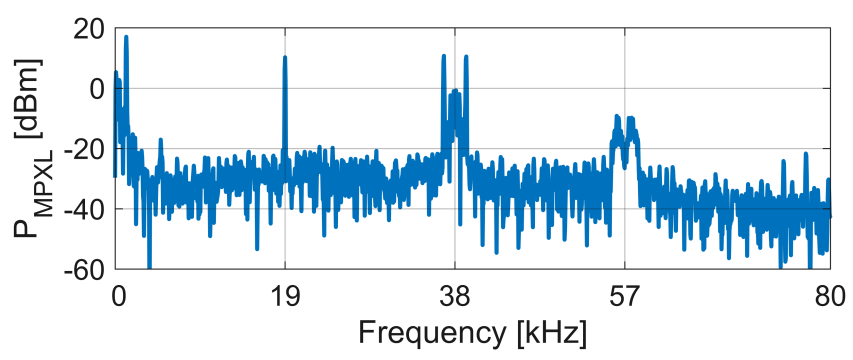

Fig. 4. Example of received high priority warning signal (baseband)

In Fig. 5, the spectrum of the received RF signal in which, the high priority local warning signal is predominant. More precisely, this spectrum is obtained in the same conditions as for Fig. 4. In our model, the local warning FM baseband signal was low pass filtered at $100 \mathrm{kHz}$ in order to respect the FM standards. This signal was then translated around $1 \mathrm{MHz}$ carrier.

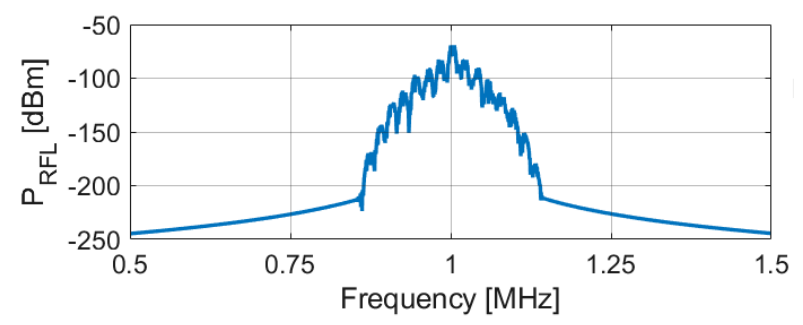

Fig. 5. Spectrum of the received RF signal when the local warning signal is predominant. 
In Fig. 6, the bit error rate of the received RDS signal depending on the difference between the two received RF signal powers, $\Delta P_{d B}=P_{R F L}-P_{R F N}$, for different values of the received RDS signal to noise ratio is plotted. The power of the received high priority local warning signal, $P_{R F L}$, depends on the distance between the car's aerial and the transmitter's antenna and may be estimated by using the equation (1).

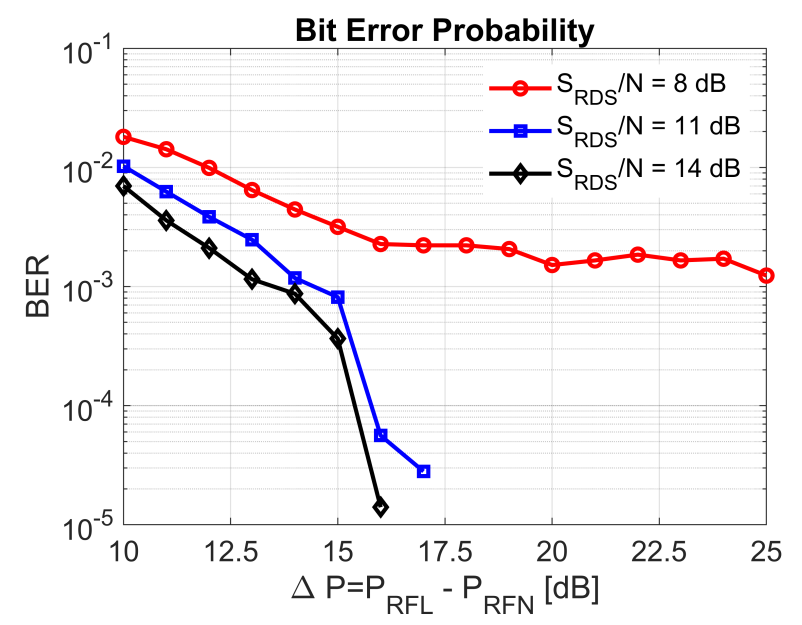

Fig. 6. BER of the received RDS signal depending on the difference between the two received RF signal powers $\Delta P$ for different values of the received RDS signal to noise ratio.

From Fig. 3 and Fig. 6, can be estimated the distance between the local FM emitter and the car's aerial at which the RDS component of the local warning signal can be received without errors. Indeed, consider a transmission of the local warning signal with an $S_{R D S} / N=11 \mathrm{~dB}$. According to Fig. 6 , in this particular case, the power level of $s_{R F L}(t)$ need to be $17.5 \mathrm{~dB}$ higher than the power level of $s_{R F N}(t)$ in order to receive the RDS component without errors. Moreover, by taking into account the average power levels of $s_{R F N}(t)$ (Table I), the following distances can be estimated, as function of the propagation environment (Table II).

\begin{tabular}{|c||c|c|c|}
\hline Area & Rural & Urban & Large cities \\
\hline Distance [meters] & 145 & 36 & 14 \\
\hline
\end{tabular}

DiSTANCE BETWEEN THE LOCAL FM EMITTER AND THE CAR'S AERIAL GUARANTEEING AN RDS TRANSMISSION WITHOUT ERRORS

These estimated values are available for an $E I R P=10$ $\mathrm{dBm}$ and if no error correction code was implemented after the RDS signal demodulation. In order to increase the transmission range for $s_{R F L}(t)$, either the EIRP can be increased, either error correction codes, decreasing the $\Delta P$ value for which no error occur, can be implemented.

In Fig. 7 is given an example of a text message, received by the high priority local warning station in the vicinity of a rail crossing. This message is decoded in Matlab Simulink from the RDS component of the $s_{R F L}(t)$ signal and may be featured on the auto-radio's display. Moreover, the corresponding warning pictogram, software built at the receiver stage, may be displayed on the car's Tablet PC.

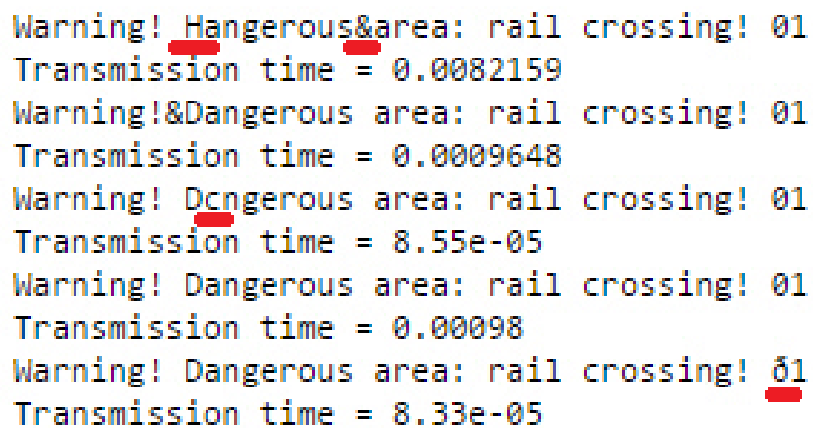

Fig. 7. Text message extracted from the RDS component of the $s_{R F L}(t)$ signal.

\section{CONCLUSION}

The paper presents a strategy to warn drivers from imminent traffic events. By using local FM emitters and the RDS component, codes corresponding to warning pictograms are sent. Software defined radio receivers coupled with tablet PC are demodulating, decoding and then displaying the corresponding pictogram. Moreover, in order to study the coexistence of such kind of local FM transmissions with the national or regional ones, this paper propose a method to estimate the local FM transmission range, exempted from errors. This method employs a simple propagation channel model and is based on the average received power levels of the national/regional FM transmissions. Future work will be dedicated to implement in practice this driver's warning scenario.

\section{REFERENCES}

[1] Rob Decae, Frouke Hermens (SWOV), "European Commission (2020) Road safety targets - Monitoring report June 2020. European Road Safety Observatory. Brussels, European Commission, Directorate General for Transport." Nov. 2020.

[2] R. G. Bozomitu, F. D. Hutu, and N. De Pinho Ferreira, “Drivers' Warning Application Through Image Notifications on the FM Radio Broadcasting Infrastructure," IEEE Access, vol. 9, pp. 13 553-13 572, 2021.

[3] D. Gettman, "DSRC and C-V2X: Similarities, Differences, and the Future of Connected Vehicles," Kimley Horn, Jun. 2020. [Online]. Available: https://www.kimley-horn.com/ dsrc-cv2x-comparison-future-connected-vehicles/

[4] "EN 62106:2001 - Specification of the radio data system (RDS) for VHF/FM sound broadcasting in the frequency range from 87,5 to 108,0 MHz," Tech. Rep., 2014.

[5] "BS.450-4 : Transmission standards for FM sound broadcasting at VHF," ITU-R, Tech. Rep., 2019.

[6] "BS.412-9 Planning standards for terrestrial FM soundbroadcasting at VHF," ITU-R, Tech. Rep., 1998.

[7] Constantine A. Balanis, Antenna theory analysis and design , 3rd ed. John Wiley and Sons, Inc., 2005.

[8] R. W. Stewart, K. W. Barlee, and D. S. Atkinson, Software defined radio using MATLAB and Simulink and the RTL-SDR, S. A. Media, Ed., 2015.

[9] "RDS/RBDS and RadioText Plus (RT+) FM Receiver," Matlab, Tech. Rep., 2020. [Online]. Available: https://www.mathworks.com/help/comm/ ug/rds-rbds-and-radiotext-plus-rt-fm-receiver.html 